\title{
Pressure-Flow Relationship and Longitudinal Distribution of Pulmonary Vascular Resistance in Heartworm-Infected Dogs
}

\author{
Motoki KONDO $^{1)}$, Makoto WASHIZU ${ }^{1) *}$, Yoshihito MATSUKURA ${ }^{1)}$, Tsukimi WASHIZU ${ }^{1)}$, Katsuyuki MIYASAKA ${ }^{2)}$ \\ and Masao TAKATA ${ }^{2)}$ \\ ${ }^{1)}$ Research Laboratory of Veterinary Medical Teaching Hospital, Nippon Veterinary and Animal Science University, 1-7-1 Kyonan-cho, \\ Musashino-shi, Tokyo 180-0023 and ${ }^{2}$ Pathophysiology Research, National Children's Medical Research Center, 3-35-31 Taishido, \\ Setagaya-ku, Tokyo 154-0004, Japan
}

(Received 9 December 2002/Accepted 8 May 2003)

ABSTRACT. The pressure-flow relationships and the longitudinal distributions of pulmonary vascular resistance in normal and heartworminfected (HWI) dogs were compared in an isolated, blood perfused preparation. The pulmonary circulation was partitioned into pulmonary arterial, middle, and venous segment based on the concept of a five element lumped model. The pulmonary arterial pressure-flow relationships were found to be non-linear and convex to the pressure axis in both normal and HWI lungs. The pressure-flow relationships of the pulmonary arterial and venous segment were linear and these slopes in the HWI lungs were significantly higher than the normal lungs. The pressure gradient of the middle segment was increased as flow increased at lower flow range, however, it was not increased during higher perfusion range in both lungs. At higher flow, the pressure gradient of the middle segment in the HWI lungs was significantly higher than the normal lungs. These results suggest that the ohmic resistance was almost equal to the sum of the two slopes of the pressure-flow relationships of the pulmonary arterial and venous segment because the pressure gradient of the middle segment was not altered as flow increased during higher perfusion rate. Because the slopes of the pressure-flow relationships of the pulmonary arterial and venous segment were increased with heartworm infection, the ohmic resistance of HWI lungs would be higher than normal lungs. The intercept pressure on the pressure axis of the linear portion of the pulmonary arterial pressure-flow relationship, a critical closing pressure, was regarded as pressure gradient of the middle segment during higher perfusing rate because the intercept pressures of pressure-flow relationships of pulmonary arterial and venous segment were almost equal to zero. Therefore, the critical closing pressure of HWI lungs would be higher than normal lungs. The pulmonary hypertension of filariasis appears to be due to an increase in ohmic resistance and elevated critical closing pressure.

KEY WORDS: canine, filariasis, pressure-flow relationship, pulmonary vascular resistance, vascular occlusion technique.

J. Vet. Med. Sci. 65(9): 965-970, 2003

In normal dogs, an increase in pulmonary blood flow is associated with a decrease in pulmonary vascular resistance without a marked elevation in pulmonary arterial pressure $[14,15]$. The reduction in pulmonary vascular resistance is generally considered to be due to capillary recruitment and/ or dilatation $[6,19]$. In dogs with heartworm disease, however, the reduction in pulmonary vascular resistance that accompanies an increase in cardiac output is smaller in magnitude than normal dogs $[12,13]$. The capillary bed in heartworm-infected (HWI) dogs may not be able to recruit and/or dilate efficiently with the increase in cardiac output $[14,15]$. The behaviors of the pulmonary vasculature in HWI dogs in response to increases in cardiac output have not been well studied.

In this study, pressure-flow curves at different perfusing flows were obtained in isolated, blood-perfused, normal and HWI canine lung lobe preparations. In addition, arterial and venous occlusion techniques were used at each perfusing flow to compare the longitudinal distributions of pulmonary vascular resistance in normal and HWI lungs [2-4, 16]. Evaluation of pressure-flow curve and distribution of pul-

\footnotetext{
* Correspondence to: Washizu, M., Research Laboratory of Veterinary Medical Teaching Hospital, Nippon Veterinary and Animal Science University, 1-7-1 Kyonan-cho, Musashino-shi, Tokyo 180-8602, Japan.
}

monary vascular resistance would allow us to understand the characteristics of vascular behavior of HWI lung in response to increased cardiac output.

\section{MATERIALS AND METHODS}

This study was approved by the Nippon Veterinary and Animal Science University institutional animal care and use committee, and all dogs were obtained from the Tochigi Animal Center. Animal preparation of isolated left caudal lung lobes (LLL) in 10 mongrel dogs were perfused for in vitro study. Five of these dogs (weighing $10.8 \pm 2.4 \mathrm{~kg}$ ) were naturally infected with heartworms, and other 5 dogs (weighing $11.0 \pm 1.6 \mathrm{~kg}$ ) were not infected with heartworms and were used as normal dogs. All dogs were tested for heartworm infection prior to experiment by wet blood smear for microfilaria and enzyme-linked immunosorbent assay (ELISA). Necropsy was performed at the end of the experiment and the number of heartworms was counted. Dogs that have variable pulmonary vascular lesions of heartworm infection were grouped as HWI by measuring pulmonary vascular resistance (PVR) during preparation that fits in a certain range of PVR. Lower or higher PVR dogs were excluded from this study and the number of heartworm infection or pathological changes of pulmonary vasculature 
were not considered.

Dogs were anesthetized with thiopental sodium $(25 \mathrm{mg} /$ $\mathrm{kg}$ IV), intubated, and ventilated by a constant volume ventilator (Kimura Medical Instruments, KV-1 +I, Tokyo). After median sternotomy, an electromagnetic flow probe (Nihon Kohden Corp., FR- I OOT, FR- I 20T, Tokyo) was placed around the ascending aorta just above the aortic valve to monitor cardiac output (CO). Stiff fluid-filled catheters were placed in the pulmonary artery and the left atrium to measure mean pulmonary arterial pressure (MPAP) and left atrial pressure (LAP). Pulmonary vascular resistance (PVR) was calculated by the following formula.

$$
\begin{aligned}
\mathrm{PVR}= & (\mathrm{MPAP}-\mathrm{LAP}) / \mathrm{CO} / \mathrm{kg} \text { body weight } \mathrm{mmHg} / \mathrm{m} l / \\
& \mathrm{min} / \mathrm{kg}
\end{aligned}
$$

Isolated perfused lung lobe: The isolated lung lobe preparation has been described previously [4]. In brief, after measuring PVR, each dog was heparinized (200 U/kg, iv) and approximately $350 \mathrm{~m} l$ of arterial blood was removed via the catheters in the femoral and carotid arteries to prime an extracorporeal perfusion system as illustrated in Fig. 1. Immediately after the exsanguination, bolus of thiopental sodium was injected and the heart and the lung were removed from the chest cavity. Plastic cannulas (ID $5 \mathrm{~mm}$ ) were placed in the LLL artery and vein to prepare the isolated, perfused preparation. In the HWI lung preparation, heartworms were removed as possible from the LLL artery before the arterial cannula was placed. It took approximately $5 \mathrm{~min}$ from bleeding blood collection for priming the perfusion system to reperfusion.

Blood from the LLL vein was drained into a downstream double compartment reservoir. The first compartment was always filled with blood during the experiment, and excess blood overflowed into the second compartment. Blood in the second compartment was pumped by a roller pump (Tonokura lka Kogyo Co., Ltd., Tow nok single pump DCH, Tokyo) through a heat exchanger $\left(38.5^{\circ} \mathrm{C}\right)$ to an upstream reservoir. Blood from the upstream reservoir flowed into the LLL artery by hydrostatic pressure and flow was monitored with an electromagnetic flow probe (Nihon Kohden Corp., FF-050T, Tokyo). The blood column height of the

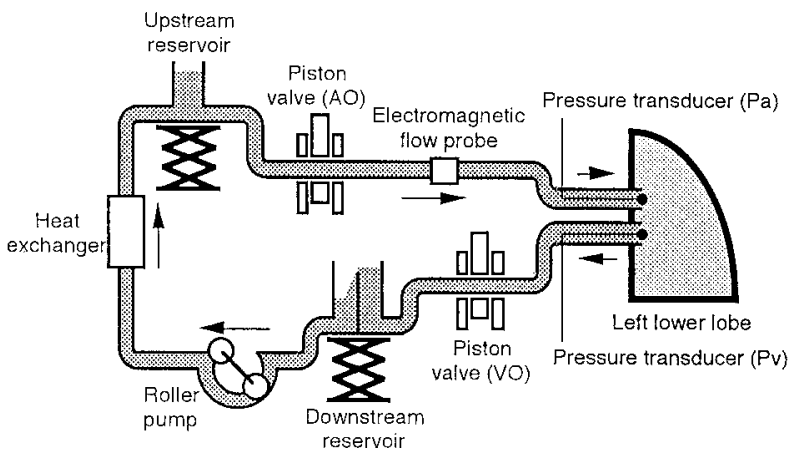

Fig. 1. A schematic illustration of the extracorporeal perfusion system used for the isolated canine lung lobe preparation. upstream reservoir was held constant by manually changing the speed of the roller pump. The height of the upstream and the downstream reservoirs relative to the LLL were adjusted to control pulmonary arterial $(\mathrm{Pa})$ and venous pressures $(\mathrm{Pv})$, both of which were measured with catheters inserted into side ports of the extracorporeal perfusion circuit. The catheters were connected to pressure transducers (Viggo Spectramed, DTX, Oxnard, CA), and Pa and Pv traces were recorded by a polygraph system (Nihon Kohden Corp., RMP-6018M and WS-682G, Tokyo).

Arterial and venous occlusion: When a steady state was achieved, arterial (AO) and venous occlusion (VO) were accomplished by rapidly closing (1ess than $20 \mathrm{~ms}$ ) piston valves that were placed just proximal to the arterial cannula and just distal to the venous cannula (Fig. 1). The vascular occlusion technique is a method for analysis of resistance distribution based on a five element lumped model [4] (Fig. 2 ). Since the AO instantaneously stopped blood flow into the pulmonary arterial segment, there was an instantaneous equilibration of pressure between the point of occlusion and the pulmonary arterial capacitor (Pca). Thus, immediately after $\mathrm{AO}$, the $\mathrm{Pa}$ rapidly equilibrated with Pca. The pressure gradient across the pulmonary arterial segment $(\mathrm{Pa})$ was calculated as Pa minus Pca. Similarly, since the VO instantaneously stopped the outflow from the pulmonary venous segment, there was an instantaneous equilibration of pressure between the point of occlusion and the pulmonary venous capacitor $(\mathrm{Pcv})$. The pressure gradient across the pulmonary venous segment $(\mathrm{Pv})$ was calculated as $\mathrm{Pcv}$ minus $\mathrm{Pv}$. A remaining pressure gradient across the middle segment (Pm) was calculated by Pca minus Pcv. The total pressure gradient across the lung lobe (APt) was calculated as Pa minus Pv. Prior to each occlusion, ventilation was stopped at end-expiration so that the lung was held at zone 111 conditions, i.e., the alveolar capillary pressure was

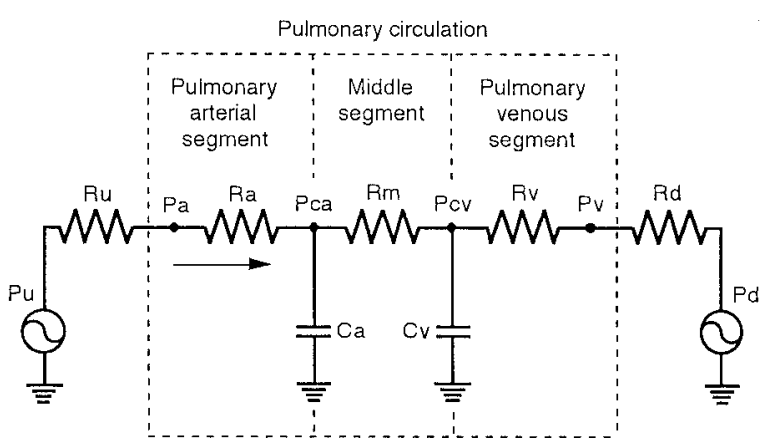

Fig. 2. An electrical analog of the five-element lumped model utilized in this study. Details of the model were described previously [4]. $\mathrm{Pu}, \mathrm{Pa}, \mathrm{Pv}$, and $\mathrm{Pd}$ are upstream, pulmonary arterial, pulmonary venous, and downstream pressures, respectively. Pca is the pressure at the pulmonary arterial capacitor $(\mathrm{Ca})$ placed at the distal end of pulmonary arterial segment. Pcv is the pressure at the pulmonary venous capacitor (Cv) placed at the proximal end of pulmonary venous segment. $\mathrm{Ru}, \mathrm{Ra}, \mathrm{Rm}, \mathrm{Rv}$, and $\mathrm{Rd}$ are upstream, pulmonary arterial, middle, venous, and downstream resistance, respectively. 


\section{Normal lung}

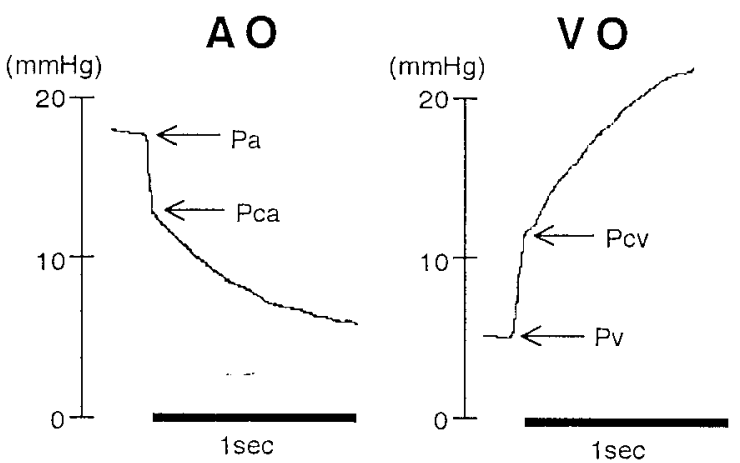

\section{Heartworm-infected lung}
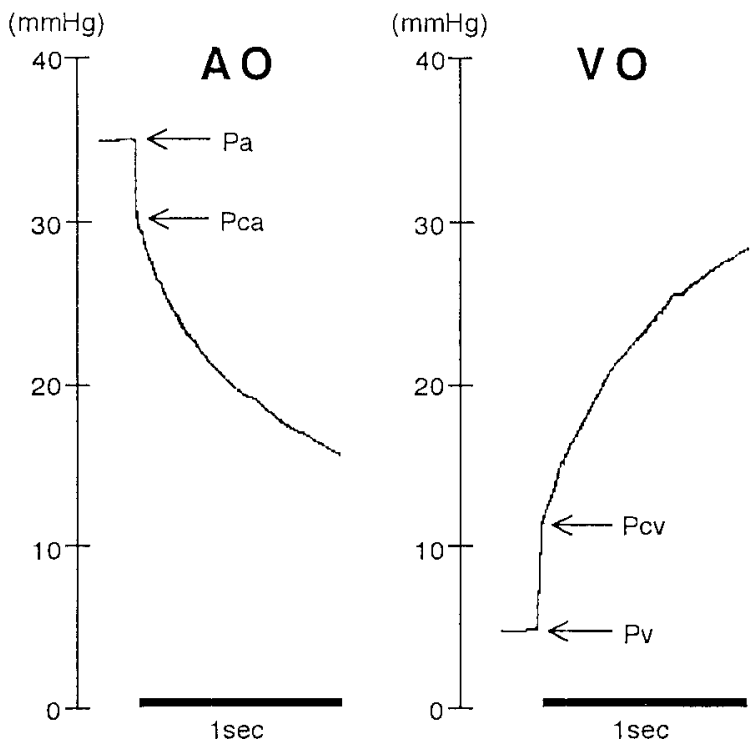

Fig. 3. Tracings of pulmonary arterial pressure $(\mathrm{Pa})$ with arterial occlusion (AO) and venous pressure (Pv) with venous occlusion (VO) from normal lung (the upper panel) and heartworminfected lung preparations (the lower panel). Pca is the pressure at the pulmonary arterial capacitor. Pcv is the pressure at the pulmonary venous capacitor.

greater than the alveolar pressure [18].

Experimental protocols: In both normal and HWI lung lobe preparations, the perfusion rate was altered from $2.5,5$, and $10,15,20$ to $25 \mathrm{ml} / \mathrm{min} / \mathrm{kg}$ body weight by changing the height of the upstream reservoir while a constant $\mathrm{Pv}$ of 5 $\mathrm{mmHg}$ was maintained. After a steady state was achieved during each perfusion rate, $\mathrm{AO}$ and $\mathrm{VO}$ were performed and the values of $\mathrm{Pa}$. Pca, Pcv and Pv were obtained. These data were used to calculate $\mathrm{Pa}, \mathrm{Pm}, \mathrm{Pv}$ and $\mathrm{Pt}$ to construct pres- sure-flow diagrams.

During the experiment, the dog did not feel any pain since the thiopental sodium was constantly administered and bolus of thiopental sodium was added to reach the level of euthanasia before the heart lung preparation was took place.

Data analysis: All results are expressed as means d: SD. Statistical analyses were made by analysis of variance (ANOVA) and Scheffe's test with a value of $p<0.05$ considered to be significant.

\section{RESULTS}

In the normal dogs, the PVR was $0.059 \pm 0.019 \mathrm{mmHg}$ / $\mathrm{m} l / \mathrm{min} / \mathrm{kg}$. In the HWI dogs, the PVR prior to removing heartworms was $0.147 \pm 0.046 \mathrm{mmHg} / \mathrm{ml} / \mathrm{min} / \mathrm{kg}$. After the heartworms were removed, PVR decreased to $0.116 \pm 0.038$ $\mathrm{mmHg} / \mathrm{min} / \mathrm{kg}$, which was still higher $(\mathrm{p}<0.05)$ than the normal dogs. At necropsy, 12 to 77 heartworms were found in the heart and the pulmonary arteries in the HWI dogs.

$P a$ and $P v$ tracings with $A O$ and VO: Figure 3 shows actual pressure tracings during $\mathrm{AO}$ and $\mathrm{VO}$ at a perfusion rate of $25 \mathrm{ml} / \mathrm{min} / \mathrm{kg}$ in normal and HWI dogs. In both groups, immediately following $\mathrm{AO}$, traces of pulmonary arterial pressure fell from $\mathrm{Pa}$ to Pca, and then decreased exponentially. Similarly, immediately following VO, traces of pulmonary venous pressure rose from $\mathrm{Pv}$ to Pcv, and then increased exponentially. No difference between the normal and the HWI dogs in terms of pressure waveform was shown. Pressure-flow relationships: Figure 4A-4D illustrated the pressure-flow diagram of $\mathrm{Pt}, \mathrm{Pa}, \mathrm{Pm}$, and $\mathrm{Pv}$ at different perfusion rates. When perfusion rate was increased at constant venous pressure, APt increased non-linearly in both normal and HWI lungs (Fig. 4A).

$\mathrm{Pa}$ and $\mathrm{Pv}$ increased linearly as the perfusion rate increased in both groups (Fig. 4B and 4D). In contrast, Pmflow relationship showed considerable non-linearity at low perfusion rates, but became more linear at higher flows in both groups (Fig. 4C). When the perfusion rate was increased over $10 \mathrm{ml} / \mathrm{min} / \mathrm{kg}$, APm was maintained at 3.2 $3.4 \mathrm{mmHg}$ in the normal lungs and at I 1.7-12.6 $\mathrm{mmHg}$ in the HWI lungs. The Pm-flow relationship contributed to the non-linearity of the Pt-flow relationships in both groups.

Longitudinal distribution of pulmonary vascular resistance: The Pt values in the HWI lungs were significantly higher $(\mathrm{p}<0.05)$ than the normal lungs at all perfusion rates (Fig. 4A). At higher flow, Pa, Pm, and Pv in the HWI lungs were significantly higher $(\mathrm{p}<0.05)$ than in the normal lungs (Fig. 4B, 4C, and 4D). Figure 5 illustrates the values of $\mathrm{Pa}$, $\mathrm{Pm}$, and $\mathrm{Pv}$ in the normal and the HWI lungs. When the perfusion rate was $2.5 \mathrm{ml} / \mathrm{min} / \mathrm{kg}$, the major resistive region to pulmonary flow was the middle segment $(\mathrm{Pm})$ in both normal and HWI lungs. When the perfusion rate was increased, the ratio of APm decreased gradually, and the ratios of $\mathrm{Pa}$ and $\mathrm{Pv}$ increased inversely in both groups. When the perfusion rate was $25 \mathrm{ml} / \mathrm{min} / \mathrm{kg}$, the major resistive regions in the normal lungs were the arterial $(\mathrm{Pa})$ and venous segments $(\mathrm{Pv})$, but the middle segment was the major resistive region 
A
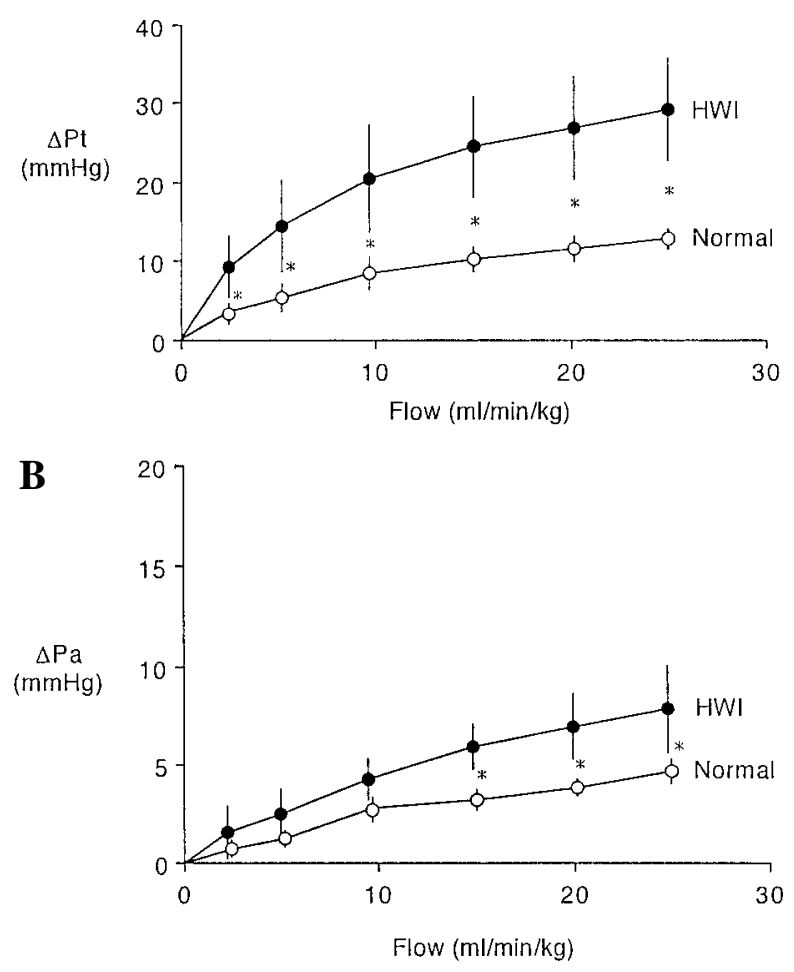

C

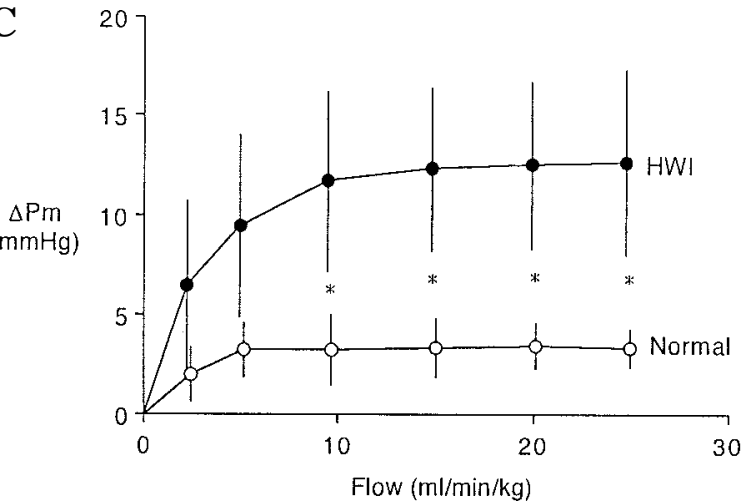

D

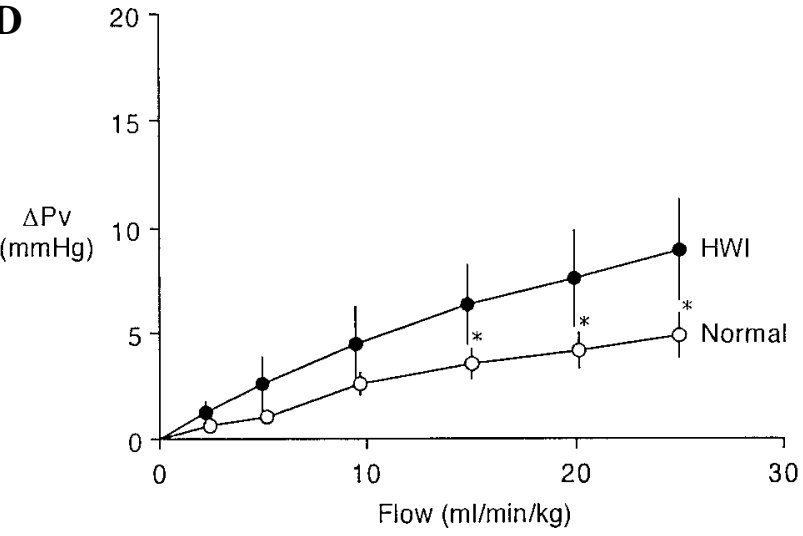

Fig. 4. The relationships of $\Delta \mathrm{Pt}$-flow (4A), $\Delta \mathrm{Pa}$-flow (4B), $\Delta \mathrm{Pm}$-flow (4C), and $\Delta \mathrm{Pv}$-flow (4D) obtained from normal (Normal, $\mathrm{n}=5$ ) and heartworm-infected lungs (HWI, n=5). Zero-flow point was not obtained experimentally. Because Pv was always set higher than alveolar pressure, we assumed that all pressure gradients $(\Delta \mathrm{Pt}, \Delta \mathrm{Pa}, \Delta \mathrm{Pm}$, and $\Delta \mathrm{Pv})$ became zero at zero flow [10]; $*=\mathrm{p}<0.05 \mathrm{HWI}$ vs. Normal.

in the HWI lungs. The significantly higher value of Pt in the HWI lungs was mainly attributed to increased Pm.

\section{DISCUSSION}

In this study, they were demonstrated that 1) non-linearity of the APt-flow relationship was attributed to a non-linear APm-flow relationship in normal and HWI lungs, and that 2) the increased pressure gradient across the middle segment was the main contributor to the leftward shift of the graph representing the APt-flow relationship in HWI lungs. The data suggest that the middle segment is a key to an understanding of the characteristic behavior of HWI pulmonary vasculature.

The usefulness of the five element lumped model has been proven in normal lung $[2-4,16]$ to analyze the longitudinal distribution of vascular resistance. However, this model has not been applied to HWI lungs. In this study, the traces of $\mathrm{Pa}$ with $\mathrm{AO}$ and $\mathrm{Pv}$ with VO could have approximated between the normal and the HWI lungs. The results suggest that this three-segment model [4] can be used to understand the longitudinal distribution of pulmonary vascular resistance in both normal and HWI lungs. Many investigators have pointed out the non-linearity of the pressure-flow relationships in normal pulmonary vascular bed, particularly at lower flow rates $[1,7,8,17]$. Around the physiological rates associated with normal arterial and venous pressures, the pressure-flow relationship is linear or slightly curvilinear [8, 17]. However, the pressure-flow relationship in HWI lung has not been well studied [11]. The Pt-flow curves obtained in this study from both normal and HWI lungs were not linear at low flow rates and became linear at high flow rates. The non-linearity of the pressureflow relationship because of capillary recruitment and/or dilatation [6, 19] might be attributed curvature of the APmflow relationship not only in normal but also HWI lungs. In another study, when cardiac output was increased, the pulmonary arterial pressure did not change in normal dog lungs, whereas the pressure increased significantly in HWI lungs $[12,14]$. This phenomenon was considered as due to reduced vascular recruitment in HWI lungs [12, 14]. In our experiment, APm did not changed as flow increase at high flow rates in both normal and HWI lungs. This phenomenon occurred because the middle segmental resistance to flow was almost zero when the capillary bed was completely recruited and/or dilated at the higher perfusion flows. Thus, capillary recruitment and/or dilatation appear to occur completely in HWI lungs at higher flow rates, but higher pressure is required for flow to occur recruitment and/or dilatation. The shape of the pulmonary arterial pres- 


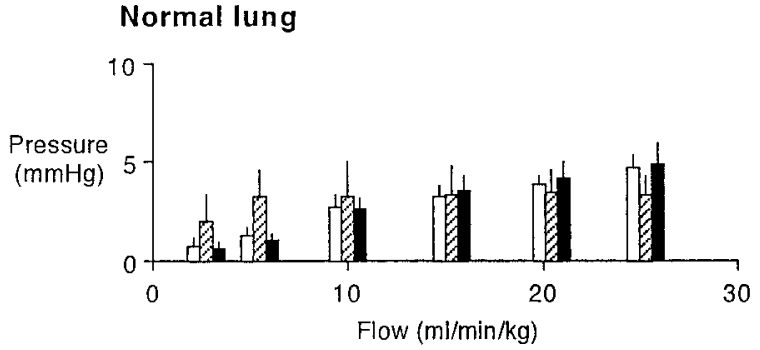

Heartworm-infected lung

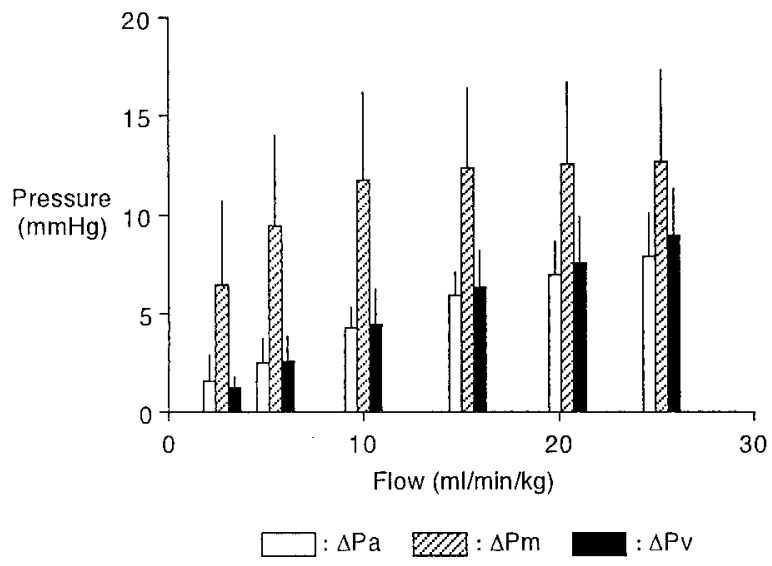

Fig. 5. $\Delta \mathrm{Pa}, \Delta \mathrm{Pm}$, and $\Delta \mathrm{Pv}$ in normal $(\mathrm{n}=5)$ and heartworminfected lungs $(n=5)$.

sure-flow curve can be expressed by the ohmic-Starling resister model $[5,9]$. In this model flow is governed by a critical closing pressure (Pcrit) and a constant, flow- and pressure-independent ohmic resistance (Rohm). Because Pcrit will act as the effective downstream pressure to flow [16], inflow pressure can be determined by Pcrit and Rohm under constant-flow conditions. The Pcrit would be a result of the forces around the vessels, such as the alveolar pressure on the pulmonary capillaries in zone II conditions [18]. If a high degree of vasomotor tone was present, the Pcrit could be higher than alveolar or outflow pressures, even in zone III conditions $[8,16]$. In pulmonary hypertension with normal outflow pressure, increased pulmonary arterial pressure would result from either elevation of Pcrit or an increase in Rohm, or both. Depending on which mechanism is predominant, there will be major differences in the way pulmonary arterial pressure responds to increased cardiac output. If the pulmonary arterial pressure was elevated as a result of higher Pcrit, pulmonary flow could increase without any major increase in pulmonary arterial pressure. If, however, the pulmonary hypertension was due to an increase in Rohm, any increase in flow would be associated with large increments of pulmonary arterial pressure [8]. The Rohm of the Pt-flow curve obtained in this study could be calculated from the linear portion in higher flow range as illustrated in Fig. 6. The slope of this linear portion of the

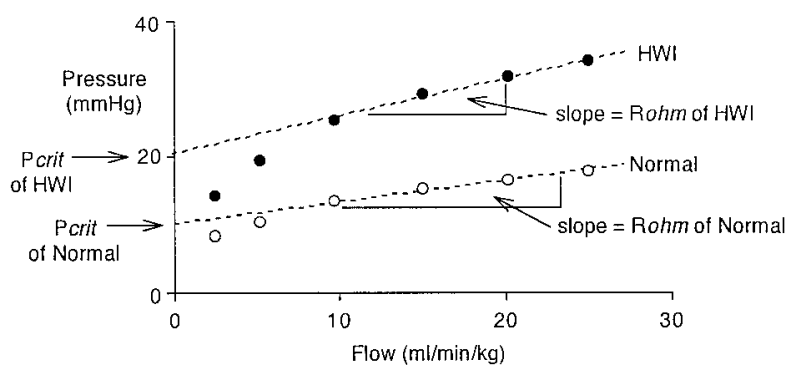

Fig. 6. The ohmic resistance (Rohm) is indicated by the slope of the linear portion (dashed-line) of the pulmonary arterial pressure-flow relationship in both normal (Normal, $n=5$ ) and heartworm infected lungs (HWI, $n=5)$. The intersection on the pressure axis of the dashed-line is equal to the critical closing pressure (Pcrit)

pressure-flow curve would be Rohm. The slope was almost equal to the sum of the two slopes of Pa- and Pv-flow curves because Pm was not changed during higher perfusion rates. The results of this study demonstrated the slopes of the Paand the Pv-flow relationships were increased with heartworm infection, and suggested that Rohm of the HWI lung was higher than normal lung. The extrapolation of the linear portion of the pulmonary arterial pressure-flow curve to zero flow produced an intercept on the pressure axis that was equal to the Pcrit as illustrated in Fig. 6 [5]. The linear portion of the pulmonary arterial pressure-flow curve consisted of the sum of the linear portions of $\mathrm{Pa}-, \mathrm{Pm}-$ and $\mathrm{Pv}-$ flow curves. Because the intercept pressures on the pressure axis of the linear portions of Pa-and Pv-flow curves were nearly equal to zero, the intercept pressure of the linear portion of Pm-flow curve could be regarded as Pcrit. The intercept pressures of the linear portions of APm-flow curves were almost equal to the pressure at higher flow range, i.e., $3.2-3.4 \mathrm{mmHg}$ in normal and $11.7-12.6 \mathrm{mmHg}$ in HWI lungs. The Pcrit of the HWI lung would be higher than the normal lung. These data suggested that the pulmonary hypertension in HWI lung was due to increases in Pcrit and Rohm. When the HWI dogs are in resting condition, the pulmonary arterial pressure might already be higher because of raised Pcrit. When the HWI dogs were excited or given a certain amount of exercise, the pulmonary arterial pressure would increase markedly because of the increased Rohm. In the clinical situation, restricted exercise is important to avoid further increases in pulmonary arterial pressure due to increased cardiac output. During anesthesia, cardiac output should be maintained to keep the pulmonary vasculature open since HWI dogs have higher Pcrit. If pulmonary arterial blood pressure dropped because of decreased cardiac output due to the negative inotropic effects of anesthetic agents, the pulmonary vasculature might collapse and serious hypoxemia will develop. In conclusion, we have compared the pressure-flow relationships and the longitudinal distributions of pulmonary vascular resistance between normal and HWI lungs. The pulmonary arterial pressure-flow relationships were non-linear in both normal and HWI 
lungs. At higher flows, the pressure gradients of all vascular segments in the HWI lungs were significantly higher than in the normal lungs. Pulmonary hypertension observed in HWI dogs appears to be due to an increase in Rohm and elevated Pcrit.

\section{REFERENCES}

1. Fishman, A. P. 1963. Dynamics of the pulmonary circulation. pp. 1667-1743. In: Handbook of Physiology, sec 2, Circulation, vol. 2 (Hamilton, W. F. and Dow, P. eds.), American Physiological Society Washington, D.C.

2. Hakim, T. S., Dawson, C. A. and Linehan, J. H. 1979. Hemodynamic responses of dog lung lobe to lober venous occlusion. J. Appl. Physiol. 47: 145-152.

3. Hakim, T. S., Michel, R. P. and Chang, H. K. 1982. Partitioning of pulmonary vascular resistance in dogs by arterial and venous occlusion. J. Appl. Physiol. 52: 710-715.

4. Kondo, M., Washizu, M., Matsukura, Y., Kobayashi, K., Motoyoshi, S., Miyasaka, K. and Takata, M. 1994. Analysis of longitudinal distribution of pulmonary vascular resistance by use of a five element lumped model. J. Vet. Med. Sci. 56: 7176.

5. Linehan, J. H., Haworth, S. T., Nelin, L. D., Krenz, G. S. and Dawson, C. A. 1992. A simple distensible vessel model for interpreting pulmonary vascular pressure-flow curves. J. Appl. Physiol. 73: 987-994.

6. Maseri, A., Caldini, O., Howard, P., Joshi, R. C., Permutt, S. and Zierler, K. L. 1972. Determinants of pulmonary vascualr volume: Recruitment versus distensibility. Circ. Res. 31: 218228.

7. McFadden, E. R. Jr. and Braunwald, E. 1984. Cor pulmonale and pulmonary thromboembolism. pp. 1572-1604. In: Heart Disease. A Textbook of Cardiovascular Medicine, 2nd ed. (Braunwald, E. ed.), W. B. Saunders Co., Philadelphia.
8. McGregor, M. and Sniderman, A. 1985. On pulmonary vascular resistance: The need for more precise definition. Am. J. Cardiol. 55: 217-221.

9. Mitzner, W. 1983. Resistance of the pulmonary circulation Clin. Chest. Med. 4: 127-137.

10. Murray, J. F., Karp, R. B. and Nadel, J. A. 1969. Viscosity effects on pressure-flow relations and vascular resistance in dogs' lungs. J. Appl. Physiol. 27: 336-341.

11. Rawlings, C. A. 1980. Acute response of pulmonary blood flow and right ventricular function to Dirofilaria immitis adults and microfilariae. Am. J. Vet. Res. 41: 244-249.

12. Rawlings, C. A. 1980. Cardiopulmonary function in the dog with Dirofilaria immitis infection: During infection and after treatment. Am. J. Vet. Res. 41: 319-325.

13. Rawlings, C. A. 1981. Exericse in the dog with Dirofilaria immitis infection. Am. J. Vet. Res. 42: 2057-2060.

14. Rawlings, C. A. 1986. Cardiopulmonary disease development. pp. 41-55. In: Heartworm Disease in Dogs and Cats. Philadelphia, W. B. Saunders Co.

15. Rawlings, C. A., McCall, J. W. and Lewis, R. E. 1978. The response of the canine's heart and lungs to Dirofilaria immitis. J. Am. Anim. Hosp. Assoc. 14: 17-32.

16. Rock, P., Patterson, G. A., Permutt, S. and Sylvester, J. T. 1985. Nature and distribution of vascular resistance in hypoxic pig lungs. J. Appl. Physiol. 59: 1891-1901.

17. Shoukas, A. A. 1975. Pressure-flow and pressure-volume relations in the entire pulmonary vascular bed of the dog determined by two-port analysis. Circ. Res. 37: 809-818.

18. West, J. B., Dollery, C. T. and Naimark, A. 1964. Distribution of blood flow in isolated lung; relation to vascular and alveolar pressures. J. Appl. Physiol 19: 713-724.

19. West, J. B., Schneider, A. M. and Mitchell, M. M. 1975. Recruitment in networks of pulmonary capillaries. J. Appl. Physiol. 39: 976-984 